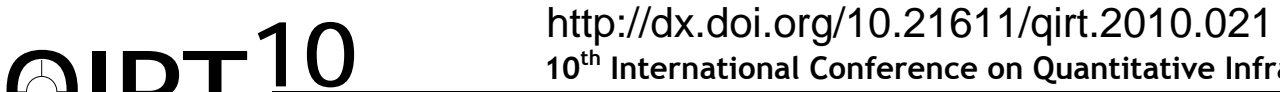 \\ $10^{\text {th }}$ International Conference on Quantitative InfraRed Thermography \\ July 27-30, 2010, Québec (Canada)
}

\section{Development of models to extract ambient variables influence from outdoor thermographic inspections}

by E.C. Bortoni*, L. Santos**

*ISEE, Itajubá Federal University, Brazil, bortoni@ieee.org

** Furnas Centrais Elétricas, Brazil, laertesa@furnas.com.br

\begin{abstract}
Infrared thermography is widely applied to power systems in the power substations and in the overhead transmission lines condition monitoring, aiming at continuous operation and maintenance purposes. As applied in an outdoor environment, the themographyc inspection is influenced by a number of ambient variables such as wind, solar radiation, humidity, and so forth. The aim of this paper is to present the development of mathematical models to extract the influence of ambient variables that can influence the diagnostic of a power system component based on thermographic inspection.
\end{abstract}

\section{Introduction}

Infrared (IR) thermal inspection is a valuable tool to determine the operating conditions of substation components. Problems such as high resistance contacts, short- and open- circuits, inductive heating, harmonics, load imbalance and overloads can often be detected through IR thermal inspections. Applications of such technology to power systems are presented since the sixties [1-2].

Despite thermal inspection seems to be a simple task, there are a number of limitations and exogenous factors of influence that can conduct to erroneous diagnosis and eventually impede the failure detection [3]. Low emissivity of the components under inspection, load current variation and small dimensions of the inspected object located at large distances are examples of drawbacks that must be overcame in an IR thermal inspection. Environmental quantities such as the solar radiation, atmospheric attenuation, wind speed, precipitation, and environment temperature and humidity variation are uncertainty factors that must be added when inspecting uncovered substations.

The works of Madding and Lyon [4] and Snell [5] consider the loading conditions and environmental components influence of IR thermal inspections. Lyon Jr. ET alii [6] evaluate the relationship between the current loading and the temperature rise in a faulty connector. In addition, the papers discusses about the limitations of techniques of condition evaluation based only in the absolute temperature or in the temperature raise, which can conduct to wrong diagnosis.

This work tries to contribute with the understanding of the influence of environmental and technical quantities over the IR thermal inspection, by presenting actual data obtained in field and developing mathematical models that allows not only to consider the environmental influences over the results of a thermal inspection, but also to remove the effect of some of these quantities to forecast the system behavior when operating in specific conditions of interest. Therefore, the determination of the expected component temperature under extreme load conditions, environment temperature and other factors of influence becomes possible.

\section{Factors of influence}

There are a number of factors that can cause influence when conducting IR inspections in an uncovered environment, as in a power substation. Despite the IR inspection seems to be a simple task, the neglecting of some factors of influence can conduct to erroneous condition evaluation of the equipment under analysis. The development of an acclimatized chamber to perform studies on this matter is subject of an accompanion paper. The main factors of influence are described as follows [7]. 


\subsection{Load current}

Despite load current isn't an ambient factor of influence, it is interesting to extract the influence of the load current to realy understand the influence of ambient variables. Therefore, the influece of ambient variables would be the difference of the target recorded temperature to the temperature rise due to the load current.

Bortoni et al [8] have developed a model to understand, to correct and to extract the temperature rise due to the load current, which is the so-called auto-regressive model.

$$
\Delta \theta_{t}=a_{0} \cdot I_{t-0 \cdot \Delta t}^{2}+a_{1} \cdot I_{t-1 \cdot \Delta t}^{2}+\ldots+a_{n} \cdot I_{t-n \cdot \Delta t}^{2}
$$

Therefore, the temperature rise due to load current depends not only of the present running current, but also of the current $(I)$ that has runned $n$ previous time intervals $(\Delta t)$. By knowing the value of the equation coefficients $\left(a_{i}\right)$, it becomes possible to recognize the temperature rise for any given current.

\subsection{Environment temperature}

The temperature of a target varies with the ambient temperature for sure. Nevertheless, the influence o environment temperature can be simply neglected if the analysis is caried out considering the temperature rise to the ambient temperature. On the other hand, it turns the detection of a defective device more difficult, mainly when the temperature rise due to the incipient failure is small. Figure 1 shows an example of a fauty device in comparison with a good one. Notice that the temperature difference between them is remarkable during the night (a) than along the day (b).
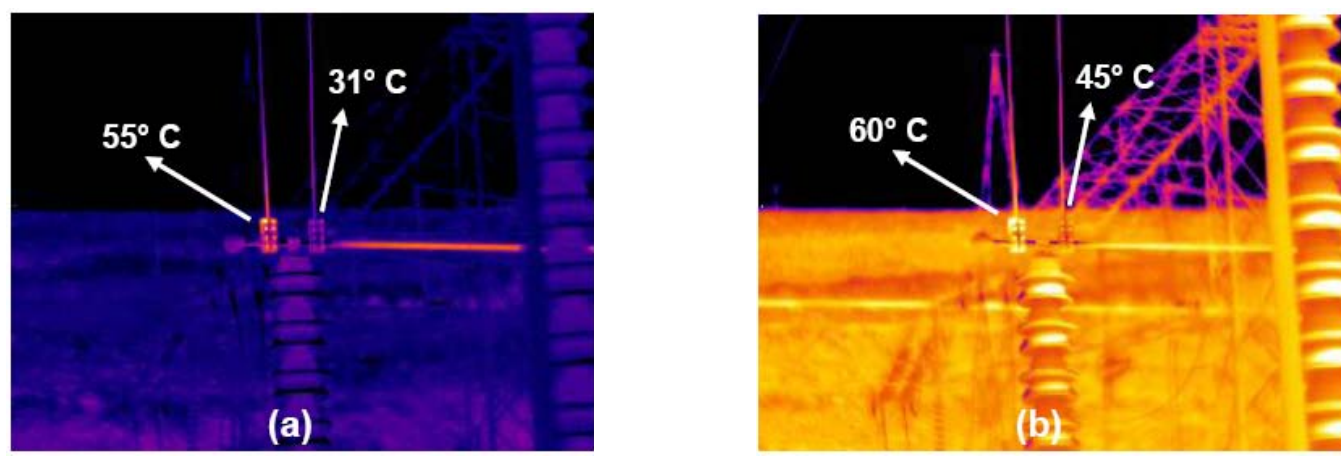

Fig. 1. Influence of the solar charge

It can be at least partially explained due to a higher absortion of a surface when compared to another one, leading to a greater temperature increase when exposed to the solar radiation.

\subsection{Solar reflection}

In general the typical components of a substation present very low emissivity for both solar and infrared radiation, mainly when they are new and polished. As their transmitance is zero for these wavelengths, the reflectance uses to be high. Therefore, the detected radiation can be, in its most part, origined in their vincinities or a reflex of solar radiation.

A current transformer of a $345 \mathrm{kV}$ substation is presented in Fig. 2. The T connection presented polished surface with low emissivity. The use of two different thermo cameras, one of 3-5 $\mu \mathrm{m}$, and other of 7.5-13 $\mu \mathrm{m}$ were carefully located in a position to get the polished surface temperature readings. Even though the regular operation of the connector, high values of temperature readings were presented due to the solar reflex. 


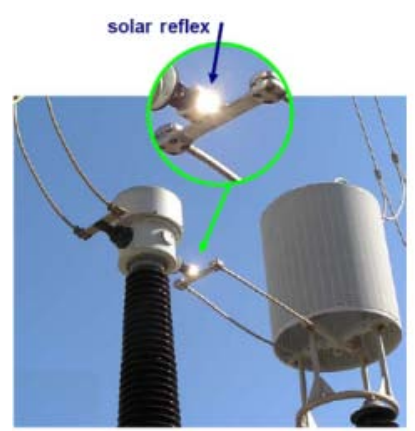

(a)

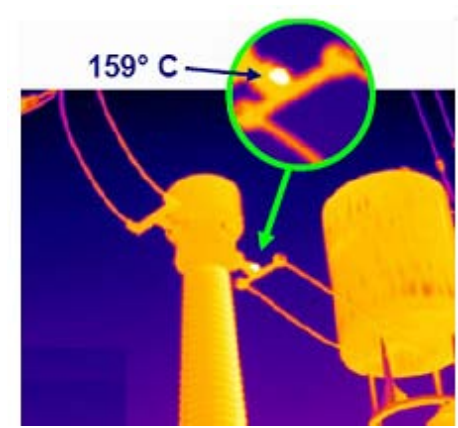

(b)

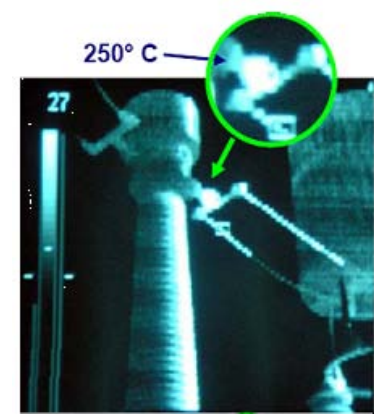

(c)

Fig. 2. Influence of the solar reflection

\subsection{Humidity}

Humidity is the concentration of water in the air. As in any substance, the air has its absortion limit called saturation. Humidity levels over the saturation limit causes fog and rain. The humidity influences thermographic surveys by cooling the component or due to attenuation as a function of the radiation dispersion. Figure 3 depicts the effects of the rain in an installation. The device temperature has reduced during the rain and got previous level only one hour later.

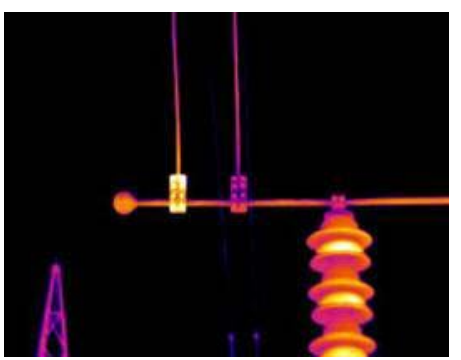

(a)

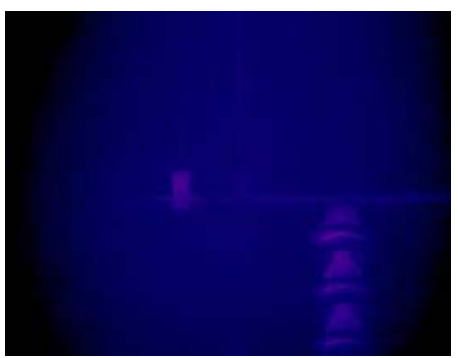

(b)

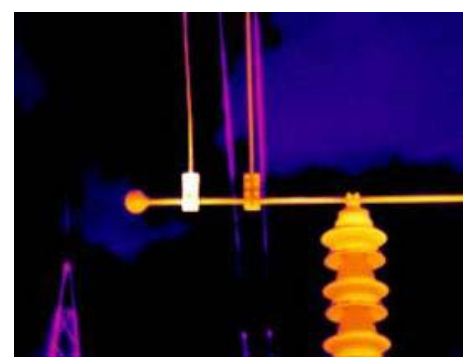

(c)

Fig. 3. Influence of humidity

\subsection{Wind}

Wind greatly affects the results of infrared thermo inspections as it increase the heat exchange with the environment, apparently reducing the real temperature of the target. Even the minor wind speed results in a great temperature decrease. Therefore, the knowledge of the wind speed influence is of utmost importance to obtain the most reliable equipment diagnostics. Figure 4 shows the influence of the wind speed in oil immersed switchgear. The temperature at null wind speed (a) is much greater than when there is a modarate wind (b).

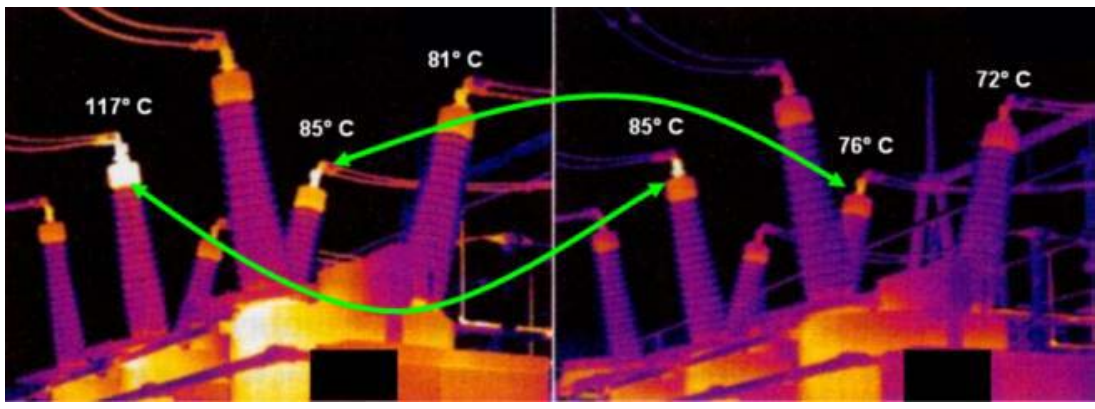

(a)

Fig. 4. Influence of wind

(b) 


\section{Mathematical modeling}

Some of the ambient factors of influence can be treated in the field. Avoidance of the solar reflection and of the inspection under rain or high humidity levels can be done by the trained thermographer. Nevertheless, there are other ambient factors of influence that can't be simply avoided in the field. The following sections, on the other hand, presents some incipient models developed to extract the influence of such factors in order to get more reliable temperature assessment and more accurate component diagnostic.

\subsection{Exponential model for the wind speed influence}

A phenomenological model of the influence of the wind over the temperature raise of a heated body is described by a decreasing exponential function of its speed. For power system components, the developed model considers that the wind speed imposes a reduction of the temperature raise due to the load condition. This reduction is described by the Reduction Factor given by

$$
F_{R}=1-k \cdot\left(1-\mathrm{e}^{-\frac{v}{v}}\right)
$$

Where $k$ and $V$ are coefficients determined for each equipment, and $v$ is the wind speed $(\mathrm{km} / \mathrm{h})$. In general value of $k$ is between 0.8 and 0.9 , while $V$ can vary from 5 to 15 .

It is important to notice that $k$ and $V$ have special physical interpretations. The $k$ coefficient expresses the maximum temperature reduction that can be imposed by the wind. Whilst for lower wind speeds the reduction of the temperature raise is very noticeable, this reduction saturates even for the strongest wind.

On the other hand, the $V$ parameter refers to the degree of influence of wind velocity on the thermal equilibrium of a homogeneous body. In analogy to a first order dynamic system, it can be known as a velocity constant, while $k$ is the gain.

Theoretically, the values of $k$ and $V$ can be obtained as

and,

$$
k=\frac{\left.\Delta \theta\right|_{v=v_{\infty}}}{\left.\Delta \theta\right|_{v=0}}
$$

$$
V=\frac{\left.\Delta \theta\right|_{v=v_{\infty}}-\left.\Delta \theta\right|_{v=0}}{d \Delta \theta /\left.d t\right|_{v=0}}
$$

Other approaches such as least squares method can also be used determine parameters for each system's component. Fig. 5 (a) and (b) present some results of the application of this formulation to data obtained in laboratory when monitoring the temperature raise for a connector when driving currents of $400(A)$ and $600(A)$, respectively.

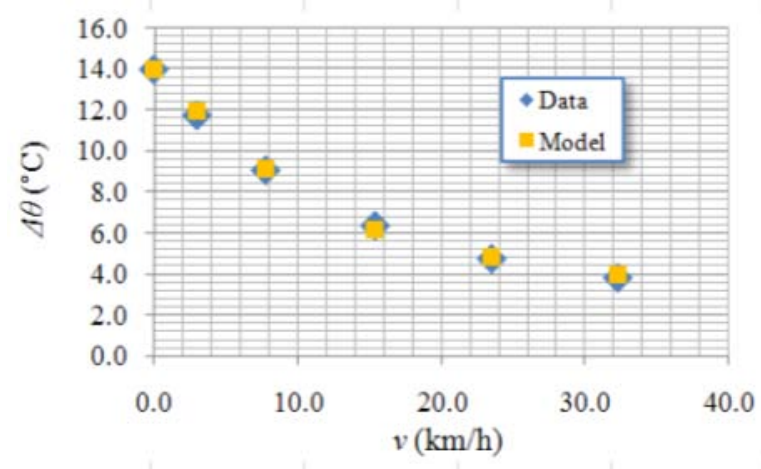

(a)

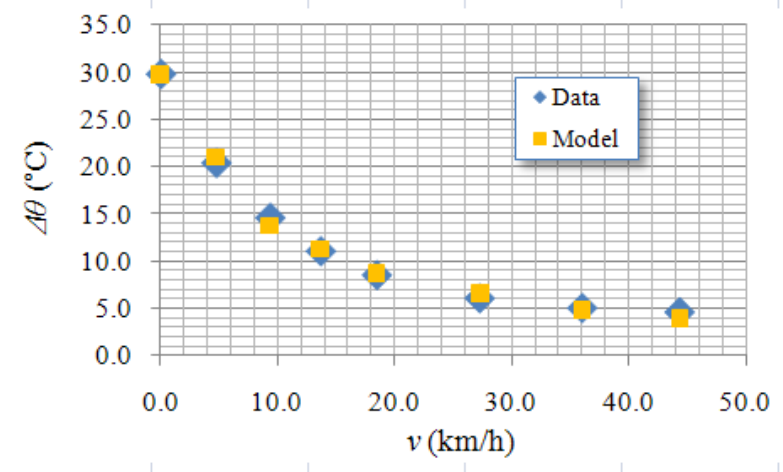

(b)

Fig. 5. Wind speed model application

The values of $k$ and $V$ were obtained 0.8 and 13.42, and 0.85 and 10.32, respectively for $400(A)$ and $600(A)$. It is important to notice that different values of $k$ and $V$ were obtained for a same device. The reason resides on the temperature raise for null wind speed. The fact is that, the higher is the temperature at null wind speed, the higher the heat transfer will be, as long as the convection mechanism of heat transfer is proportional to the temperature difference. 


\subsection{Multivariable model for factors of influence}

The main environmental factors considered in this work are the temperature, humidity, rain, wind velocity and solar radiation. A simple multivariable linear model to estimate the temperature rise over the environmental temperature is proposed:

$$
\Delta \theta=\Delta \theta_{I}+\sum_{i \in I} w_{i} \cdot x_{i}
$$

Where $\Delta \theta\left({ }^{\circ} \mathrm{C}\right)$ is the temperature rise over the environmental temperature, $\Delta \theta_{I}\left({ }^{\circ} \mathrm{C}\right)$ is the temperature rise due to the load current, obtained from the previous presented models, $I$ is the set of considered environmental factors, $w_{i}$ is the weight of influence of the $x_{i}$ environmental factor. This equation is rewritten for each instant of time allowing for the obtaining of the weight coefficients by using the least squares method.

This methodology was applied to a different set of data obtained in field in order to estimate the influence of the rain, wind velocity and solar radiation. A setup was prepared with a thermal-camera and a meteorological data acquisition system as presented in Fig. 6.

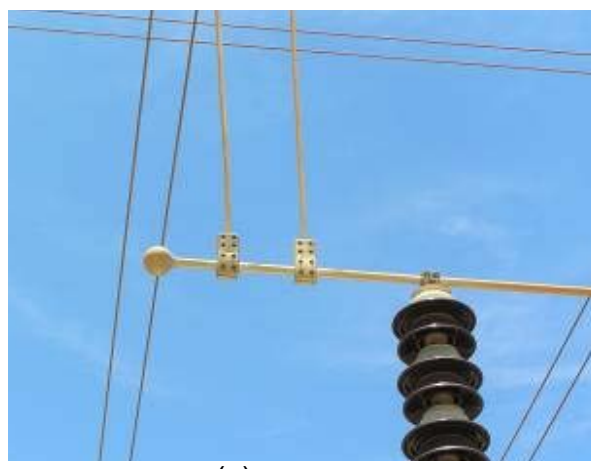

(a)

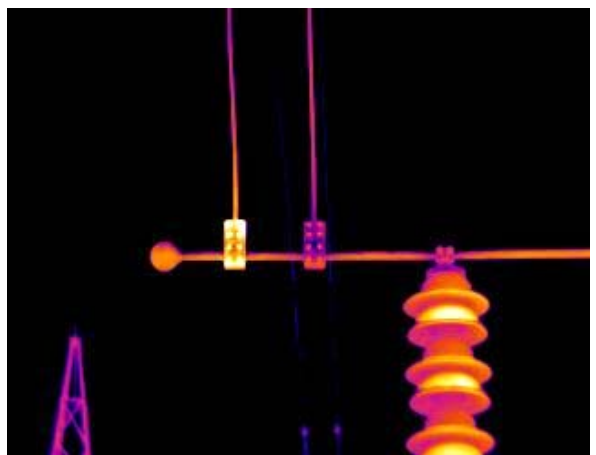

(b)

Fig. 6. Connector under analysis

The field data recorded is presented in Fig. 7. Humidity information was not used because it was observed a strong inverse correlation with the environmental temperature.

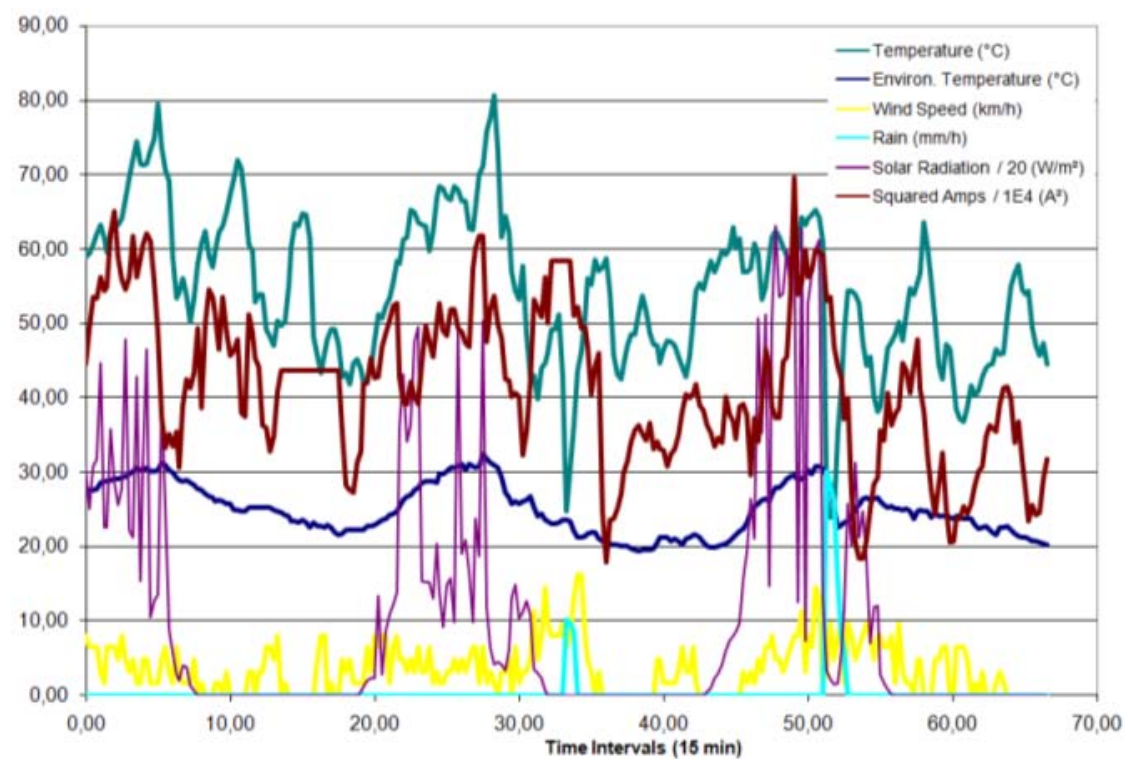

Fig. 7. Field data record 
A self correlated model was used estimate the time delay between the current and the temperature raise. The delay was found 45 minutes. Employing the presented formulation, a model was adjusted to obtain the temperature rise as a function of load current $(I)$, wind speed $\left(x_{W S}\right)$, solar radiation $\left(x_{S R}\right)$ and rain $\left(x_{R}\right)$, which is:

$$
\Delta \theta=4.906 \cdot 10^{-5} \cdot I^{2}-1.241 \cdot x_{W S}+6.626 \cdot 10^{-3} \cdot x_{S R}-1.428 \cdot x_{R}+13.079
$$

As long as this equation relates the temperature rise as a function of several quantities, it can also be employed to obtain the temperature rise for the worst conditions, i.e., maximum load current and solar radiation, and minimum wind speed and rain, as well. Figure 8 presents the estimated absolute temperatures for regular operation, which showed excellent agreement with the field obtained in field, and that calculated for extreme conditions.

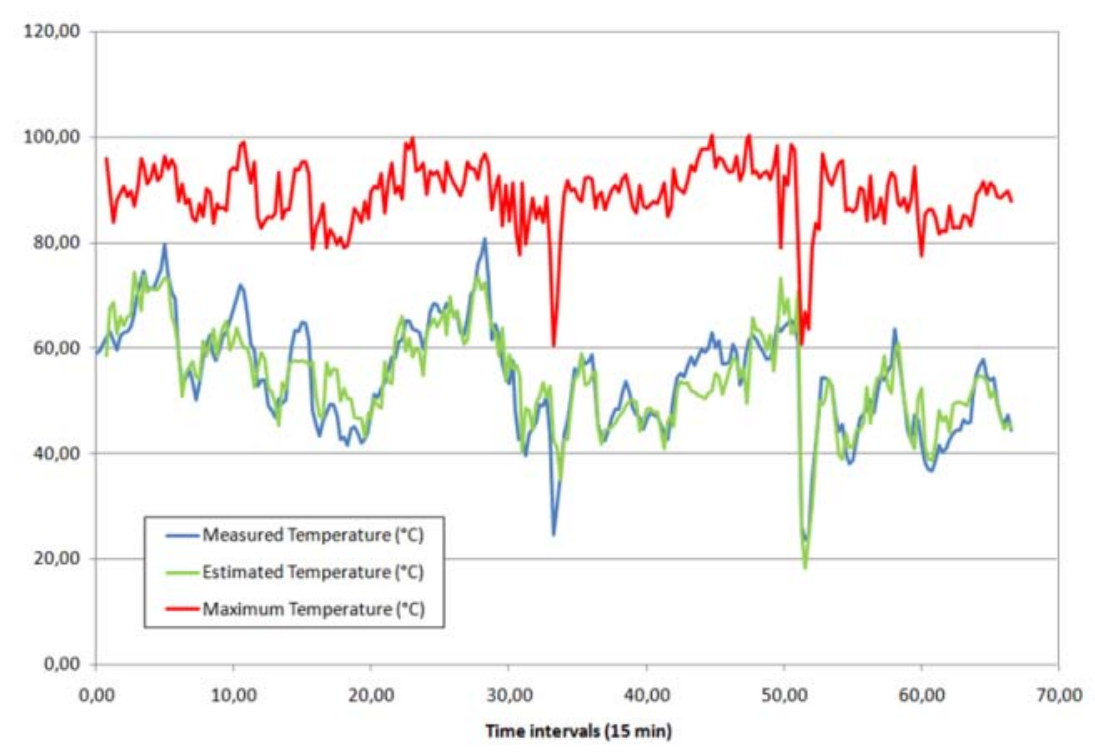

Fig. 8. Calculated temperatures for regular and extreme conditions

\section{CONCLUSIONS}

The work presented the first results of a study under development which aims at obtaining more consistent procedures for IR inspections in outdoor environments, under the influence of technical, physical and environmental factors of influence. The work makes use of an acclimatized chamber that allows simulating several environmental and loading conditions. The paper presented an auto-regressive methodology to estimate the temperature rise for the worst case, i.e., the maximum loading current, as long as traditional methods are suitable for constant load currents instead of variable load currents like that found in power systems.

The work also presented a proposal of wind influence modeling to extract its effect over outdoor thermographic inspections. A wind velocity dependent temperature reduction factor was developed and applied in order to estimate the target temperature in the absence of wind. The model was applied to data obtained in the laboratory, where an acclimatized chamber was used allowing for simulating environmental and loading conditions. The results of the developed model application showed very good agreement when compared to the laboratorial results. 


\section{ACKNOWLEDGMENT}

This work was developed under the R\&D program of FURNAS Centrais Elétricas in a partnership with Itajubá Federal University. Prof. Bortoni would like thank FAPEMIG and CNPq for the financial support of his researches.

\section{REFERENCES}

[1] G. Ferreti, A. Giorgi, A New Type of Pyrometer Employed for Preventive Maintenance in Electric Utilities, L`Energia Elettrica, N 12, 1969.

[2] C.W. Brice, Infrared detection of hot spots in energized transmission and distribution equipment, Electric Power Systems Research, Volume 1, Issue 2, April 1978, pp 127-130.

[3] J. Snell, R.W. Spring, Developing Operational Protocol for Thermographic Inspection Programs, SPIE Vol. 1682, 1992.

[4] R. Madding, B.R. Lyon Jr., Environmental Influences on IR Thermography Surveys, Maintenance Technology 1999.

[5] J. Snell, A Different Way to Determine Repair Priorities Using a Weighted Matrix Methodology, Snell Infrared 2001.

[6] B.R. Lyon Jr, G.L. Orlove, L.P. Donna, The Relationship between Current Load and Temperature for Quasi-Steady State and Transient Conditions, Infrared Training Center 2002.

[7] Santos, L.; "Termografia Infravermelha em Subestações de Alta Tensão Desabrigadas" (in Portuguese). Master Dissertation. Itaubá Federal University. 2006.

[8] Bortoni, E.C.; Santos, L.; Bastos, G.S.; Souza, L.E.; Craveiro, M.A.C.; "Extracting Load Current Influence From Infrared Thermal Inspections". IEEE Trans. on Power Delivery. Digital Object Identifier 10.1109/TPWRD.2010. 2046068. 